ten der türkischen Zyprer ${ }^{83}$ bleibt abzuwarten ${ }^{84}$, ob dann die Sitze der türkisch-zyprischen Abgeordneten erstmals seit 1963 wieder besetzt sein werden. Sollte dies der Fall sein, sollten diejenigen Punkte aus Alt-Staatspräsident Makarios' Vorschlag zur Änderung der Verfassung übernommen werden, die das Repräsentantenhaus direkt betrafen: (1) die Wahl des Präsidenten des Hohen Hauses und seines Vizepräsidenten durch alle gesetzlichen Mitglieder, Zyperngriechen und -türken; (2) die effektive Möglichkeit der Vertretung des griechisch-zyprischen Parlamentspräsidenten durch seinen türkisch-zyprischen Vize; (3) die Abschaffung der nach Bevölkerungsgruppen getrennten Abstimmungen im Repräsentantenhaus, in welchem Artikel der Verfassung sie auch vorgesehen sind ${ }^{85}$. Nur auf diese Art und Weise könnte eine effektive Zusammenarbeit im Hohen Haus eines einheitlichen Zypern funktionieren.

83 Vgl. unter anderem den Bericht der Zeitung Kathimerini vom 12. September 2008 (auf Griechisch); relevante Berichte der Gesellschaft für Zypernstudien vom 12. Oktober 2008 („Wöchentliche Treffen zu Zypern-Gesprächen vereinbart“) und 27. Oktober 2008 („Sechste Runde der Zyperngespräche“) auf http://www.cypern.de/?m=200810 (Abruf am 15. Dezember 2008) sowie die Einschätzung eines nur relativen Fortschritts in der Zeitung Kathimerini vom 18. Dezember 2008 (auf Griechisch) und Christofias' erklärtes Bestreben, die Gespräche kontinuierlich und effektiv fortzuführen, vgl. das entsprechende Grußwort von Christofias in der Zeitung Charavgivom 25. Dezember 2008 (auf Griechisch).

84 Eine zusätzliche Schwierigkeit dürfte wohl nach dem 19. April 2009 und der nunmehr effektiven Zwangsmachtteilung zwischen Sozialdemokraten und Nationalkonservativen im türkisch besetzten Norden Zyperns entstehen; vgl. unter anderem den entsprechenden Bericht der Zeitung Kathimerini vom 20. April 2009 (auf Griechisch).

85 Vgl. in diesem Zusammenhang Jörg-Detlef Kühne, a.a.O. (Fn. 8), S. 80; Iossif Iossif, a.a.O. (Fn. 2), S. 73; Konstantinos Goumas / Stelios Kotsiopoulos (Hrsg.), a.a.O. (Fn. 2), S. 980.

\title{
Die Verurteilung des Premierministers durch Urteil des thailändischen Obersten Gerichtshofs vom 21. Oktober 2008
}

\author{
Natjira Kanthong
}

Vorbemerkung der Redaktion: Das nachstehend wiedergegebene Urteil gegen den Vorgänger des jetzigen thailändischen Premiers verdient in zweifacher Hinsicht Aufmerksamkeit: Erstens politisch, weil es durchaus nicht zu einer wirklichen Beruhigung der innenpolitisch höchst angespannten Lage dort beitragen konnte. Vielmehr scheint es zur Zeit nach den politischen Dauerdemonstrationen in Bangkok nur um eine gewisse Verschnaufpause in dem Bemühen der dem Vorgänger verpflichteten so genannten Rothemden zu gehen, ihn wieder an die Macht zu bringen. Dabei bietet das vorliegende Gerichtsverfahren nur eine Facette der politisch-sozialen Transformation, in der sich Thailand nicht anders als etliche seiner Nachbarländer befindet. Begleitet von Vorwürfen der Vetternwirtschaft und des Stimmenkaufs, geht es um eine langsame Verschiebung von den angestammten alten Eliten 
hin zu einer politisch breiteren Partizipation der Bevölkerung, wobei insbesondere der Stadt-Land-Gegensatz erwähnt sei und die Tatsache, dass der aktivistische Vorgängerpremier und seine Rothemden für entsprechende Veränderungen stehen.

Zweitens ist die Entscheidung juristisch von Bedeutung, weil erfolgreiche Anklagen gegen Regierungschefs nach wie vor Seltenheitswert haben. Der hier betroffene Premier, der sich den Folgen seiner Verurteilung durch Flucht ins Ausland entzog und dessen bis dahin bestehende Parlamentsmehrheit durch Herausbrechen mehrerer Abgeordneter verloren ging, sieht sich seither in einer gewissen Märtyrerrolle. Dabei dürfte es zum Verständnis des folgenden Urteils nicht zuletzt von Interesse sein, dass bis heute die einseitige Besetzung politischer Schlüsselstellungen in Staat und Armee nach wie vor zur bedenklichen Tradition dortiger Machtausübung gehören soll, und zwar auch hinsichtlich der Richterbestellung. ${ }^{1}$

\section{Zum Sachverhalt der Entscheidung}

Während der dortigen Wirtschafts- und Finanzkrise hatte der staatliche thailändische Hilfsfonds für Finanzinstitutionen (SHF) Vermögen und Grundstücke von Banken und sonstigen Finanzinstitutionen, die durch die Finanzkrise in Not geraten waren, zu einem übermäßig hohen Preis gekauft. Sie sollten durch diese finanzielle Unterstützung ihre Geschäfte weiter fortführen können. Danach brachte der SHF das gekaufte Vermögen und die Grundstücke zur öffentlichen Versteigerung unter des Devise: je höher der Verkaufspreis, desto geringer der eigene Verlust. Das heißt, Verkäufe zu Spitzenpreisen sollten die Verluste des staatlichen Hilfsfonds verringern.

Bei einer solchen Versteigerung erwarb Potjaman Shinawatra, die Ehefrau des seinerzeitigen thailändischen Ministerpräsidenten, ein Grundstück in guter Lage Bangkoks, und zwar begünstigt zum Preis von 772 Millionen Baht (circa 370 Millionen Euro). Dabei spielte nach thailändischer Tradition die Autorität von Ranghöheren, hier des Premiers Thaksin Shinawatra, eine große Rolle. Durch seinen überwältigenden politischen Einfluss und seine Autorität konnte er Konkurrenten aus der Versteigerung verdrängen. Im Ergebnis gab es nur drei Bieter, die höchstwahrscheinlich wussten, dass sie bei dieser Versteigerung zurückstehen sollten. Aus diesem Grund konnte der staatliche Hilfsfonds bei diesem Grundstücksverkauf nicht den angemessenen Preis erzielen. Seiner Einschätzung nach hätte er bei unbeeinflusster Versteigerung das Grundstück für mindestens 870 Millionen Baht (knapp 420 Millionen Euro) verkaufen können.

Dieser offensichtliche Interessenkonflikt zwischen persönlichen und staatlichen Interessen fiel indessen nicht in einen gesetzesfreien Raum. Vielmehr ist es Regierungsbeamten und ihren Familienangehörigen seit dem im Jahre 1999 erlassenen Anti-Korruptionsgesetz (AKG) verboten, Verträge mit staatlichen Organisationen zu schließen. Es kam deshalb zu einem pressebeachteten Strafverfahren.

1 Vgl. Jochen Buchsteiner, Kleine und große Übel. Die Lage in Thailand bleibt verfahren, in: FAZ vom 18. April 2009, S. 12. 


\section{Entscheidungsergebnis und zentrale Rechtsfragen}

Die Staatsanwaltschaft erhob Anklage gegen Premierminister Thaksin Shinawatra und seine Ehefrau Potjaman wegen Amtsmissbrauchs gemäß Art. 4, 100, 122 AKG in Tateinheit mit Vorteilsannahme gemäß $\$ 152$ und Pflichtverletzung gemäß $\$ 157$ in Verbindung mit $\$ \$ 83,86,90,91$ des thailändischen Strafgesetzbuches (ThStGB). Zugleich stellte die Staatsanwaltschaft gemäß $₫ 33 \mathrm{ThStGB}$ den Antrag auf Beschlagnahme von Grundstück und Kaufpreis.

Am 21. Oktober 2008 (Az. 1/2550) verurteilte der Oberste Gerichtshof den inzwischen ins Ausland geflohenen angeklagten Premier in Abwesenheit wegen Amtsmissbrauchs gemäß Art. 100 in Verbindung mit Art. 122 des AKG zu einer Freiheitsstrafe von zwei Jahren. Seine Ehefrau wurde freigesprochen. Der Beschlagnahmeantrag hinsichtlich des Grundstücks und Kaufpreises blieb ohne Erfolg.

Die zentralen Rechtsfragen, um die es in dem Verfahren ging, waren vor allem folgende: (1) Gültigkeit des Anti-Korruptionsgesetzes von 1999; (2) Zuständigkeit des seit 2006 als Ermittlungsbehörde bestehenden „Ausschusses zur Vermögensüberprüfung“ sowie des beim Obersten Gerichtshofs eingerichteten besonderen Spruchkörpers für Regierungsmitglieder, denen Straftaten vorgeworfen werden; (3) Auslegung der Begriffe „staatliche Organisation“ und „Aufsichtspflicht“; (4) Strafbarkeit angeklagter Täter und Teilnehmer wegen Amtsmissbrauchs; (5) Strafbarkeit des angeklagten Premiers wegen Vorteilsannahme und Pflichtverletzung in Ausübung seiner amtlichen Stellung; (6) Beschlagnahme von Grundstück und Kaufpreis.

\section{Entscheidungsgründe zu den vorgenannten Rechtsfragen}

(1) Im Jahr 2006 kam es in Thailand zu einem Militärputsch und nachfolgend zur Aufhebung der damaligen Verfassung. Da das Anti-Korruptionsgesetz von 1999 als Sondergesetz aufgrund der Verfassung von 1997 verabschiedet wurde und Teil dieser Verfassung war, prüften die Richter zunächst die Rechtsgültigkeit des AKG nach Aufhebung der alten Verfassung von 1997. Diese Verfassung war durch „Erklärung des Rats für demokratische Reform (RDR) Nr. 3“ vom 19. September 2006 aufgehoben worden. Außerdem hatte das Militär die Legislative und Exekutive übernommen. Lediglich die rechtsprechende Gewalt, allerdings mit Ausnahme des Verfassungsgerichts, blieb unberührt.

Der Oberste Gerichtshof entschied sich für die Anwendbarkeit des AKG von 1999, denn er sah dessen Rechtsgültigkeit unabhängig vom Bestehen der alten Verfassung als gegeben an. Seiner Auffassung nach blieben die Gesetze gültig, bis sie vom Gesetzgeber ausdrücklich außer Kraft gesetzt würden, was bisher nicht geschehen sei. Vielmehr bestätigte der RDR mit seiner „Erklärung Nr. 19” vom 22. September 2006 die Gültigkeit des AKG von 1999 sogar ausdrücklich.

(2) Durch seine weitere „Erklärung Nr. 30“ vom 30. September 2006 war außerdem der Ausschuss für Vermögensüberprüfung (AVÜ) gegründet worden. Auch insoweit stellte sich die Frage der Verfassungsmäßigkeit. Im Falle der Verfassungswidrigkeit hätte der AVÜ aufgelöst werden müssen. Zwar gab es zu dieser Frage bereits ein früheres Urteil des Verfassungsgerichts vom 30. Juni 2008 (Az. 5/2551), das die Verfassungsmäßigkeit der „Erklärung Nr. 30" bestätigte. Doch stellte sich die weitere Frage, ob der AVÜ überhaupt 
gegründet werden durfte, da es schon eine andere ähnliche staatliche Institution, die Nationale Kommission zur Korruptionsbekämpfung (NKKB), gab, so dass sich Zuständigkeiten und Aufgaben beider Kommissionen überschneiden würden.

Das Verfassungsgericht begründete seine Entscheidung damit, dass der AVÜ nur gegen Straftaten von Politikern ermitteln dürfe, solange die Strafverfahren von der Staatsanwaltschaft vor Gericht noch nicht eingeleitet worden seien. Obwohl diese Behörde anders als Polizei und Staatsanwaltschaft keine Ermittlungstätigkeit nach der Strafprozessordung ausübe, sei der AVÜ befugt, von Politikern begangene Straftaten im Amt zu verfolgen. Ferner hätten die Übergangsverfassung von 2006 und die aktuelle Verfassung von 2007 rückwirkend die Verfassungsmäßigkeit der fraglichen „Erklärung Nr. 30“ und mithin den Ausschuss zur Vermögensüberprüfung genehmigt - mit der Folge, dass er parallel mit der NKKB seine Tätigkeit ausüben durfte. Der AVÜ war also zuständig, gegen Premier Thaksin Shinawatra und seine Ehefrau zu ermitteln.

Bleibt zur Rechtsfrage der Zuständigkeit des Obersten Gerichtshofs zu sagen, dass durch die Verfassung von 1997 ein neuer strafrechtlicher Spruchkörper für Straftaten im Amt von Regierungsbeamten geschaffen wurde. Demnach erklärte sich der Oberste Gerichtshof auch für Strafsachen gegen Politiker zuständig.

(3) Weiter war festzustellen, ob der staatliche Hilfsfonds für Finanzinstitutionen eine „staatliche Organisation“ ist. Nach dem AKG von 1999 und dem Gesetz über Bieterverfahren bei staatlichen Stellen desselben Jahres hat der Wortlaut „staatliche Organisation“ in beiden Gesetzen dieselbe Bedeutung. Der staatliche Hilfsfonds wurde von der zentralen Notenbank Thailands (ZBT) gegründet. Er ist eine Abteilung dieser Bank. Folglich gilt der SHF gemäß Art. 100 Abs. I AKG als staatliche Organisation.

Art. 100 AKG regelt ein besonderes Tätermerkmal. Das Gesetz setzt voraus, dass der Täter als Amtsträger beziehungsweise hochrangiger Politiker tätig sein muss. Die Auslegung dieser Vorschrift beschränkt sich dabei nicht nur darauf, dass der Täter die direkte Aufsicht über die Zentralbank beziehungsweise den Hilfsfonds haben muss. In der Praxis hatte der Premierminister die Aufsicht über den Hilfsfonds mittelbar durch den Finanzminister, da er als Regierungschef für den gesamten Bereich der Exekutive verantwortlich war und zwar in vertikaler Weise durch den Finanzminister auch für den SHF. Thaksin Shinawatra erfüllte mithin das Merkmal eines hochrangigen Politikers, der die Aufsicht über den Hilfsfonds führte.

(4) Gemäß Art. 100 AKG ist es verboten, dass Regierungsbeamte und ihre Familienangehörigen Verträge mit staatlichen Organisationen schließen. Evidenter Zweck dieses Gesetzes ist die Vermeidung eines Interessenkonflikts. Familienangehörige von Regierungsbeamten sind vom Tatbestand mit umfasst, da die enge Beziehung zwischen Ehegatten leicht zu Bevorzugungen führen kann. Art. 100 AKG galt folglich auch für die Ehefrau des Premiers, die in öffentlicher Versteigerung das Grundstück aus Staatsbesitz erworben hatte. Damit war Regierungschef Shinawatra gesetzlich als Vertragspartei anzusehen und wurde aus diesem Grund vom Obersten Gerichtshof für schuldig befunden. Seine bereits erwähnte Verurteilung zu zwei Jahren Haft erfolgte wegen Verstoßes gegen Art. 100 in Verbindung mit Art. 122 AKG.

Die Vorschriften des AKG von 1999 sind als Sonderstrafrecht einzuordnen. Gemäß Art. 122 bedarf es des besonderen Tätermerkmals, das heißt, dass sich eine Bestrafung gegen Politiker richten darf, gegen andere wie Ehegatten von Politikern hingegen ausgeschlossen ist. Solche Personen können nicht wegen Amtsmissbrauchs als Täter oder Mittäter bestraft 
werden. Falls für solches Handeln - wie vorliegend - die Strafbarkeit nicht ausdrücklich bestimmt wird, hat eine Verurteilung zu unterbleiben. Daher sprach der Oberste Gerichtshof die Ehefrau frei.

Es bleibt indessen zu kritisieren, dass die Entscheidung nicht erschöpfend ist. Denn obwohl Frau Shinawatra als Nicht-Politikerin wegen Amtsmissbrauchs nach dem AKG nicht als Täterin oder Mittäterin bestraft werden durfte, konnte sie nach dem Strafgesetzbuch Gehilfin des Täters sein. Aufgrund ihrer Beihilfehandlung wäre sie dann nach $₫ 86 \mathrm{ThStGB}$ zu bestrafen gewesen. Gemäß dieser Vorschrift ist für Gehilfen das Strafmaß auf zwei Drittel der Strafe zu mildern.

(5) Abgesehen von der Strafbarkeit nach dem AKG von 1999 kann ein Amtsträger sich auch gemäß $\$ \$ 152,157 \mathrm{ThStGB}$ strafbar machen. In diesem Fall ist festzustellen, ob Strafbarkeit wegen Vorteilsannahme nach $\$ 152$ und wegen Pflichtverletzung bei Ausübung des öffentlichen Amtes nach $\$ 157$ vorliegen. Laut Sachverhalt hatte Premier Shinawatra nur seine Unterschrift gegeben, um der Eigentumsübertragung des fragwürdig erstandenen Grundstücks an seine Ehefrau zuzustimmen. Solches Verhalten gilt jedoch weder als Vorteilsannahme noch als Pflichtverletzung. Daher liegt die Voraussetzung des Tatbestandsmerkmals des $\$ 152$ (Vorteilsannahme durch Dienstpflichtverletzung) nicht vor. Ferner hatte er auch nicht gemäß $\$ 157$ durch positive Pflichtausübung oder Pflichtunterlassung Dritte geschädigt; ein Verstoß gegen $\$ 152$ und $\$ 157$ ThStGB lag nicht vor. Da insofern keine Straftat des Haupttäters gegeben war, wurde seine Ehefrau nicht als Gehilfin bestraft.

(6) Gegenstände, die durch eine rechtswidrige Tat erlangt wurden, sind nach $\$ 33$ ThStGB zu beschlagnahmen. Laut Sachverhalt ist das bloße Bieten in einer Versteigerung und das Abschließen eines Kaufvertrages nicht rechtswidrig. Was strafrechtswidrig ist, ist der Amtsmissbrauch aufgrund des politischen Einflusses durch den Premierminister. Die Versteigerung und der Grundstückskaufvertrag selbst sind jedoch rechtswirksam. Folglich schied eine Beschlagnahme des umstrittenen Grundstückes und des gezahlten Kaufpreises gemäß $\$ 33$ ThStGB aus. Der Oberste Gerichtshof hat in seinem Urteil vom 21. Oktober 2008 dementsprechend entschieden und mithin der staatsanwaltlich beantragten Beschlagnahme nicht Folge geleistet. 